\title{
Weight change trends and overall survival in United States veterans with follicular lymphoma treated with chemotherapy
}

\author{
Daphne Y. Xiao, BA ${ }^{1,2}$, Suhong Luo, MPH ${ }^{1,3}$, Katiuscia O’Brian, MA ${ }^{1,3}$, Weijian Liu, MPH ${ }^{1}$, \\ and Kenneth R. Carson, MD, PhD ${ }^{1,3,4}$ \\ ${ }^{1}$ Research Service, St. Louis Veterans Affairs Medical Center, St. Louis, MO \\ ${ }^{2}$ Washington University School of Medicine, St. Louis, MO \\ ${ }^{3}$ Division of Oncology, Department of Internal Medicine, Washington University School of \\ Medicine, St. Louis, MO \\ ${ }^{4}$ Division of Public Health Sciences, Department of Surgery, Washington University School of \\ Medicine, St. Louis, MO
}

\begin{abstract}
Understanding weight change patterns in follicular lymphoma (FL) may be important for the assessment of prognosis as well as the long-term care of survivors. A retrospective cohort of United States veterans with a new diagnosis of FL between October 1, 1998 and September 30, 2010 was assembled. Weight changes were evaluated before, during, and after treatment in $896 \mathrm{FL}$ patients who received cyclophosphamide, doxorubicin, vincristine, and prednisone, with or without rituximab ( $\mathrm{CHOP}+/-\mathrm{R})$; cyclophosphamide, vincristine, and prednisone, with or without rituximab (CVP +/- R); or rituximab monotherapy. Weight decreased an average of $1.4 \mathrm{~kg}$ during therapy, and $>5 \%$ weight loss during this time period was associated with worse overall survival. Weight increased to an average of $1.4 \mathrm{~kg}$ above baseline by 24 months after treatment initiation, with $15 \%$ gaining greater than $10 \%$ of their baseline weight. Weight management strategies may be an important part of long-term survivorship planning.
\end{abstract}

\section{Keywords}

body weight changes; veterans; lymphoma

\section{INTRODUCTION}

\begin{abstract}
Weight loss $>10 \%$ in the six months prior to diagnosis, one of the B-symptoms, is a known adverse prognostic factor for non-Hodgkin lymphoma (NHL) [1]. Less well studied are weight change patterns during and after chemotherapy. We recently published a report evaluating weight change trends in a large cohort of patients with diffuse large B-cell lymphoma (DLBCL) [2]. Other reports investigating weight change trends in NHL exist, but
\end{abstract}

CORRESPONDING AUTHOR: Kenneth R. Carson, MD, PhD, Division of Oncology, Washington University School of Medicine, 660 S. Euclid Ave., Campus Box 8056, St. Louis, MO 63110, Phone: 314-362-0492, Fax: 314-747-5123, kcarson@ dom.wustl.edu. POTENTIAL CONFLICTS OF INTEREST: The authors have no relevant conflicts of interest to disclose. 
are limited by small sample size, short follow up, self-reported weights, or inclusion of multiple NHL subtypes [3-6]. There are no large studies looking at weight change trends in follicular lymphoma, the second most common subtype of NHL.

Understanding weight change patterns both during and after chemotherapy may have important clinical implications for FL patients. Weight change during chemotherapy has been associated with decreased survival in various solid tumors, and may have prognostic value in follicular lymphoma [7-10]. Long term weight change, particularly weight gain, is an important cancer survivorship issue, as obesity is associated with cardiovascular disease, diabetes, lower quality of life and poorer survival [11-15]. With many FL patients living more than 10 years after initial treatment, it is particularly important in this patient population to prevent obesity and its associated health risks [16,17].

In this study, we evaluate a cohort of United States veterans with FL diagnosed and treated within the United States Veterans Health Administration (VHA) system. The purpose of this study is to describe long-term and short-term weight change trends in FL patients, identify factors associated with long-term weight gain, and determine whether weight change during chemotherapy affects time to next treatment, overall survival, and NHL-specific survival.

\section{MATERIALS AND METHODS}

\section{Study Cohort}

A retrospective cohort of patients with a new diagnosis of FL between October 1, 1998 and September 30, 2010 with follow up until October 17, 2014 was assembled from the Veteran's Health Administration Central Cancer Registry (VACCR) based on the InterLymph classification system (International Classification of Diseases (ICD)) -O3 codes 9690, 9691, 9695, and 9698 for FL [18]. Data were obtained from all 21 VHA regions throughout the United States. Patients were excluded for the following reasons: missing vital status, diagnosis of human immunodeficiency virus (HIV), primary central nervous system involvement, primary cutaneous FL, inadequate histologic confirmation, no treatment, treatment outside of the VHA, or treatment with regimens other than cyclophosphamide, doxorubicin, vincristine, and prednisone, with or without rituximab (CHOP +/- R); cyclophosphamide, vincristine, and prednisone, with or without rituximab (CVP +/- R); or rituximab monotherapy. The study was approved by the Veterans Affairs St. Louis Health Care System and Washington University institutional review boards prior to cohort assembly.

\section{Clinical Data Collection}

Data on histologic diagnosis, date of birth, date of diagnosis, race, sex, disease stage, and the presence of systemic B-symptoms (fever $100.4^{\circ} \mathrm{F}$, weight loss $>10 \%$ of body weight in 6 months, and night sweats) were provided by the VACCR. Patient records were also linked to additional VHA administrative datasets to obtain vital sign data including height and weight, hemoglobin level, ICD-9 codes for co-morbid conditions, and date of death. Focused data abstraction was performed using the VHA Compensation and Pension Records Interchange 
software system to collect data on lactate dehydrogenase (LDH), and chemotherapy doses and dates of administration.

\section{Measurements and Definitions}

Weight at 1 year prior to treatment was defined as weight measured closest to but within + / -3 months of 1 year prior to date of first treatment. Weight at treatment initiation was defined as weight measured closest to but within 2 weeks of first treatment date. Weight at 3 months after treatment initiation was measured at the date closest to 3 months after first treatment date $+/-1$ month. Weight at 24 months after treatment initiation was measured at the date closest to 24 months after first treatment date $+/-3$ months.

Height consistently recorded at any time in the clinical history was considered accurate. Body mass index (BMI) was calculated as weight measured in kilograms divided by the square of height measured in meters $\left(\mathrm{kg} / \mathrm{m}^{2}\right)$ and were categorized in accordance with World Health Organization guidelines [19]. Both height and weight information were captured by clinical staff at the time of vital sign assessment with either inpatient and outpatient clinical encounters.

The Romano adaptation of the Charlson co-morbidity index was calculated for patients included in the study cohort using ICD-9 codes for co-morbid conditions present at the time of diagnosis [20]. LDH was dichotomized as elevated or not elevated at time of diagnoses based on local reference ranges. Hemoglobin level was dichotomized as $<12 \mathrm{~g} / \mathrm{dL}$ and $\geq 12$ $\mathrm{g} / \mathrm{dL}$. Age at diagnosis was dichotomized to $>60$ years of age and $\$ 60$ years of age for survival and logistic regression analysis and was used as a continuous variable in all other analyses. Patients were identified as having a second malignancy if a new ICD-9 code for malignancy was present on at least two inpatient or outpatient encounters following follicular lymphoma treatment initiation.

Time to next treatment was measured from the date of initiation of primary treatment to the date of initiation of second-line therapy. Time zero for landmark analysis was the first day of the seventh month after treatment initiation. Overall survival was measured from the start of the landmark analysis to the date of death. NHL-specific survival was measured from the start of the landmark analysis to the date of death due to NHL. Patients without death information were assumed to be alive at the time of last recorded death within the cohort (October 17, 2014); an assumption supported by previous studies demonstrating that $>97 \%$ of veteran death events are captured in VHA vital status files [21,22]. The ICD-9 and ICD-10 codes 200, 202, C82, C83, C84, and C85 for cause of death were used to identify death due to NHL.

\section{Statistical Analyses}

Chi-square, Cochran-Mantel-Haenszel, and student's t-test were used for univariate analyses, where appropriate. One-way ANOVA was used to evaluate differences in age, comorbidity score, and weight change by treatment type. Subsequent pairwise comparisons of age, comorbidity score, and weight change by treatment type were performed with student's t-test, using Bonferroni adjustment for multiple comparisons. Logistic regression explored the variables associated with weight gain $>10 \%$ at 24 months. Variables with p- 
value $<0.05$ on univariable logistic regression analysis were subsequently entered simultaneously into a multivariable logistic regression model. A landmark technique was used for Kaplan-Meier and Cox analyses evaluating the association between weight change at 3 months after treatment initiation and overall survival and NHL-specific survival, measuring only patients who survived at least 6 months after treatment initiation [23]. The proportional hazards assumption was tested for all Cox variables using Schoenfeld residuals and by insertion of time dependent covariates. A two-tailed a significance level of 0.05 was considered statistically significant. Logistic regression was performed using SPSS version 20. All other statistical analyses were performed using SAS version 9.2 (SAS Institute, Cary, $\mathrm{NC})$.

\section{RESULTS}

\section{Demographics}

Of the 2235 FL patients initially identified, 1077 (48\%) patients met inclusion criteria and $896(40 \%)$ had weight information at treatment initiation (Figure 1). The mean patient age at diagnosis was 63.4 years. The majority of patients were men (96\%) and Caucasian (89\%), and $73 \%$ had Stage III/IV disease. Hemoglobin level was $<12 \mathrm{~g} / \mathrm{dL}$ in $20 \%$ of patients and lactate dehydrogenase was elevated in $26 \%$ of patients. The mean Charlson co-morbidity score at diagnosis was 2.0, and the mean BMI at treatment initiation was 28 (Table I).

Most patients received CHOP $+/-\mathrm{R}$ as treatment (49\%), with 34\% receiving CVP $+/-\mathrm{R}$ and $17 \%$ receiving rituximab monotherapy. Age was significantly different across treatment groups $(\mathrm{p}<0.001)$. Patients who received $\mathrm{CHOP}+/-\mathrm{R}$ were younger than those who received CVP +/- R (61.1 years vs. 65.4 years, $\mathrm{p}<0.001)$ or rituximab monotherapy (61.1 years vs. 66.2 years, $\mathrm{p}<0.001)$. The mean Charlson co-morbidity score was also significantly different across treatment groups $(\mathrm{p}<0.001)$. Patients who received $\mathrm{CHOP}+/-\mathrm{R}$ had a lower mean co-morbidity score than those who received CVP +/- R (1.65 vs. $2.25, \mathrm{p}<0.001)$ or rituximab monotherapy (1.65 vs. 2.40, p<0.001). Stage, elevation of LDH, and hemoglobin level did not vary significantly across treatment groups.

\section{Weight Change Trends}

Sixty-six percent of patients lost weight in the year prior to treatment, with a mean and median weight change of $-2.7 \mathrm{~kg}(-2.8 \%$, SD 6.9) and $-2.3 \mathrm{~kg}(-2.5 \%)$. Thirty-four percent of patients lost $>5 \%$ of their initial weight by the time of treatment initiation.

This trend of weight loss continued during the treatment period. Mean and median weight change between treatment initiation and 3 months after treatment initiation was $-1.4 \mathrm{~kg}$ $(-1.5 \%$, SD 6.0$)$ and $-0.5 \mathrm{~kg}(-0.6 \%)$, with a majority of patients losing weight $(56 \%)$ and $23 \%$ of patients losing $>5 \%$ of their weight between the start of chemotherapy and 3 months after treatment initiation. In contrast, by 24 months after treatment initiation, most patients experienced weight gain. The mean and median long-term weight change from treatment initiation to 24 months after treatment initiation was $+1.4 \mathrm{~kg}(+1.8 \%$, SD 8.7$)$ and $+1.5 \mathrm{~kg}$ $(+1.8 \%)$, with a majority of patients $(59 \%)$ gaining weight and $15 \%$ of patients gaining $>10 \%$ of their baseline weight after treatment completion. 
Short-term weight changes differed by treatment type. Mean weight change between treatment initiation and 3 months after treatment initiation was $-1.9 \mathrm{~kg}(-2.0 \%$, SD 6.8), $-1.2 \mathrm{~kg}(-1.4 \%$, SD 5.5), and $-0.1 \mathrm{~kg}(-0.1 \%$, SD 4.3$)$ for patients treated with $\mathrm{CHOP}+/-$

$\mathrm{R}, \mathrm{CVP}+/-\mathrm{R}$, and rituximab monotherapy, respectively. Patients treated with CHOP-based therapy experienced more weight loss from treatment initiation to 3 months after treatment initiation than those treated with rituximab monotherapy ( $\mathrm{p}=0.004)$. However, there was no significant difference in weight change between treatment groups by 24 months after treatment initiation $(\mathrm{p}=0.78)$. Mean weight change between treatment initiation and 24 months after treatment initiation was $+1.4 \mathrm{~kg}(2.1 \%$, SD 9.3), $+1.5 \mathrm{~kg}(1.8 \%$, SD 7.9), and $+0.8 \mathrm{~kg}(0.9 \%, \mathrm{SD} 8.0)$ for patients treated with $\mathrm{CHOP}+/-\mathrm{R}, \mathrm{CVP}+/-\mathrm{R}$, and rituximab monotherapy, respectively.

In the 24 months following treatment initiation, $23.6 \%$ of patients received second-line chemotherapy. Patients who did not receive second-line chemotherapy gained significantly more long-term weight than those who did (mean weight change of $+1.8 \mathrm{~kg}$ versus $-0.2 \mathrm{~kg}$ from treatment initiation to 24 months after treatment initiation, $\mathrm{p}=0.016$ ).

\section{Factors Associated with Long-term Weight Gain}

In order to identify the clinical variables associated with significant weight gain at 24 months after treatment initiation, a logistic regression analysis was performed on patients with weight information from one year prior to treatment, at treatment, at 3 months after treatment initiation, and at 24 months after treatment initiation $(n=404)$. In the multivariable model, weight loss $>5 \%$ in the year prior to treatment (Odds Ratio (OR) 7.12, 95\% Confidence Interval (CI) 3.54 to 14.33), weight gain between 0 to $5 \%$ during treatment (OR $3.64,95 \%$ CI 1.66 to 8.00 ), and weight gain $>5 \%$ during treatment (OR 9.11, 95\% CI 3.51 to 23.63 ) were associated with increased risk of weight gain $>10 \%$ at 24 months, compared with weight gain or loss $\mathbf{5 \%}$ (in the year prior to treatment), and weight loss during treatment, respectively (Table II).

\section{Weight Change and Survival}

At the end of the follow-up period, 285 patients died. The most common cause of death was death due to non-Hodgkin lymphoma (47\%), followed by death due to other malignancies $(13 \%)$, cardiovascular disease (11\%), and lung disease (2\%).

A subset of patients who were treated prior to September 30, 2005 with follow-up until September 30, $2010(n=299)$ were examined to evaluate the incidence of second malignancy. In this subset, 40 (13\%) patients developed a second malignancy following treatment for follicular lymphoma. There was no association between weight loss $>5 \%$ during treatment and subsequent development of second malignancy (log-rank $\mathrm{p}=0.21)$.

Kaplan-Meier survival analysis of all patients demonstrated that weight loss $>5 \%$ during treatment was associated with decreased time to next treatment $(\mathrm{p}=0.033$, Figure $2 \mathrm{~A}$ ) compared to weight gain or loss $\mathbf{5} \%$. Analysis of patients living at least 6 months after treatment initiation demonstrated that weight loss $>5 \%$ during treatment was associated with decreased overall survival $(\mathrm{p}<0.001)$ and NHL-specific survival $(\mathrm{p}=0.005)$ compared to weight gain or loss $\mathbf{5} \%$. Overall survival at 5 years was $53 \%$ for patients who lost $>5 \%$ of 
weight at three months after treatment initiation, compared to $70 \%$ for patients with $\mathbf{5 \%}$ loss or weight gain at three months after treatment initiation (Figure 2B). NHL-specific survival at 5 years was $72 \%$ for patients who lost $>5 \%$ of weight at three months after treatment initiation, compared to $83 \%$ for patients with $\mathbf{5 \%}$ loss or weight gain at three months after treatment initiation (Figure 2C).

A Cox proportional hazards model assessing the association between weight loss during treatment and survival while controlling for age, disease stage, comorbidities, elevated LDH, B symptoms, hemoglobin level, BMI at treatment initiation, and treatment type showed that weight loss $>5 \%$ during treatment was independently associated with increased risk of second-line treatment initiation (Hazard Ratio (HR) 1.52, 95\% CI 1.17 to 1.98), increased mortality (HR 1.75, 95\% CI 1.35 to 2.26) and increased NHL-specific mortality (HR 1.58, 95\% CI 1.09 to 2.30). Additional variables included in the multivariate survival models are shown in Table III.

\section{DISCUSSION}

This is the first large study evaluating short- and long-term weight changes in the FL population following treatment with $\mathrm{CHOP}+/-\mathrm{R}, \mathrm{CVP}+/-\mathrm{R}$, or rituximab monotherapy. We found that the average patient initially experiences weight loss during treatment, followed by weight gain in the 24 months after treatment initiation. Weight loss during treatment was associated with worse overall survival and NHL-specific survival compared to weight gain or weight maintenance during treatment. Risk for substantial long-term weight gain was highest in patients who experienced weight loss $>5 \%$ in the year prior to treatment as well as those who experienced weight gain during treatment.

FL patients lost a mean of $1.4 \mathrm{~kg}$ between treatment initiation and 3 months after treatment initiation. Weight change during treatment varied by treatment type, and patients who received CHOP-based therapy experienced more weight loss during treatment than those who received CVP-based therapy or rituximab monotherapy. The magnitude of weight loss in FL patients receiving CHOP-based therapy is similar to that of our previous study in DLBCL patients receiving CHOP-based therapy $(-1.9 \mathrm{~kg}$ in FL patients versus $-2.0 \mathrm{~kg}$ in DLBCL patients)[2]. This suggests that weight loss during therapy may be driven more by treatment-related factors than disease-related factors.

Weight loss $>5 \%$ during treatment was associated with a $17 \%$ decrease in overall survival, compared to weight gain or loss $\mathbf{5} \%$. Several previous studies have examined postdiagnosis weight changes in NHL patients and their effect on survival, with conflicting results. A study of 573 Connecticut women with NHL reported that both weight loss and weight gain after diagnosis were associated with worse survival compared to weight maintenance [3]. In contrast, a study of 30 Serbian patients with NHL found that weight gain after diagnosis was associated with improved survival compared to either weight loss or weight maintenance, as did a study of 138 Taiwanese NHL patients [4,5]. These studies included multiple NHL subtypes, making it difficult to compare their results with our study of only FL patients. Unlike these studies, we did not observe weight gain during treatment to be associated with either worse or improved survival compared to weight maintenance. 
Evaluation of weight loss at the end of treatment may have important clinical value. The Follicular Lymphoma International Prognostic Index (FLIPI), based on clinical characteristics obtained at the time of diagnosis, is widely used for risk assessment in FL but fails to identify patients with a particularly poor outcome [24,25]. Assessment of dynamic variables such as weight at multiple time points could lead to more accurate and individualized prognostication. Weight change could also be used to inform subsequent treatment approaches. Given that weight loss varies by treatment type, patients who experience significant weight loss during initial therapy could be considered for novel and less intensive treatment, such as immunotherapy, should they relapse and require additional lines of treatment.

Following treatment completion, FL patients gained an average of $1.4 \mathrm{~kg}$ over 24 months, which is significantly higher than the rate observed in a healthy, similarly aged population of men ( $0.37 \mathrm{~kg}$ over 24 months) [26]. The patients at highest risk for significant long-term weight were those who lost $>5 \%$ weight in the year prior to treatment and gained $>5 \%$ weight during treatment. This is consistent with our previous study of weight change in DLBCL patients and suggests that lifestyle interventions and health behavior counseling should be a part of survivorship care planning in the FL population.

There are multiple strengths of this study. First, the VHA provided a large study cohort with comprehensive clinical data drawn from patients diagnosed and treated throughout the United States. Second, weight measurements were objectively measured and recorded for clinical care purposes, reducing potential reporting biases. Third, patients had an equal opportunity for inclusion regardless of comorbidities or other factors that may introduce selection bias observed in studies of patients enrolled in clinical trials. Finally, the VHA has detailed and accurate records on overall survival.

There are also limitations that should be noted. First, the study cohort was comprised almost entirely of men, which may limit our ability to extrapolate these findings to women. Second, we were unable to assess how the observed weight changes were related to changes in body composition. Futures studies are warranted to assess long and short-term body composition changes in FL patients receiving chemotherapy. Finally, the VHA patient population has a lower median income than that of the general population, which could result in more extreme average weight gain after treatment completion given the inverse relationship between income and obesity previously observed in the United States [27].

\section{CONCLUSION}

Evaluation of weight change trends before, during, and after CHOP +/- R, CVP +/- R, and rituximab monotherapy in patients with follicular lymphoma demonstrated that patients experience weight loss before and during treatment, followed by weight gain after treatment completion. Among FL patients who survived to 24 months after therapy, long-term weight gain was greatest in those who lost a significant amount of weight prior to treatment as well as those who gained weight during treatment. These patients could be targeted for physical activity and nutritional counseling at the time of treatment completion. Finally, weight loss during treatment is associated with decreased overall survival and NHL-specific survival. 
Weight loss during treatment could be considered in conjunction with other dynamic variables, such as PET activity [28], to assess prognosis at the end of therapy and guide subsequent treatment approaches.

\section{Acknowledgments}

This work was supported by: The NCCN Young Investigator Awards (YIA), The Barnes-Jewish Hospital Foundation, The American Cancer Society (MSRG-13-077-01-CPHPS), the National Cancer Institute at the National Institutes of Health (U54CA155496 and 5K12HL087107), and the Washington University Institute of Clinical and Translational Sciences grant UL1TR000448, sub-award TL1TR000449, from the National Center for Advancing Translational Sciences of the National Institutes of Health. The content is solely the responsibility of the authors and does not necessarily represent the official views of: The United States Department of Veterans Affairs, the National Cancer Institute, or the National Institutes of Health.

\section{References}

1. Dewys WD, Begg C, Lavin PT, et al. Prognostic effect of weight loss prior to chemotherapy in cancer patients. Am J Med. 1980; 69:491-497. [PubMed: 7424938]

2. O'Brian K, Luo S, Ganti A, et al. Short- and Long-term weight changes among United States veterans with diffuse large B-cell lymphoma treated with CHOP chemotherapy. Leuk Lymphoma. 2015 Jul.25:1-7.

3. Han X, Stevens J, Bradshaw PT. Body mass index, weight change, and survival in non-Hodgkin lymphoma patients in Connecticut women. Nutr Cancer. 2013; 65:43-50. [PubMed: 23368912]

4. Chin Y, Liu J, Tai J, et al. The significance of body weight change in non-Hodgkins lymphoma. Anticancer Res. 1999; 19:5607-5610. [PubMed: 10697626]

5. Stanisavljevic NS, Marisavljevic DZ. Weight and body composition changes during R-CHOP chemotherapy in patients with non-Hodgkin's lymphoma and their impact on dose intensity and toxicity. J BUON. 2010; 15:290-296. [PubMed: 20658724]

6. Lynce F, Pehlivanova M, Catlett J, Malkovska V. Obesity in adult lymphoma survivors. Leuk Lymphoma. 2012; 53:569-574. [PubMed: 21888618]

7. Thivat E, Thérondel S, Lapirot $\mathrm{O}$, et al. Weight change during chemotherapy changes the prognosis in non metastatic breast cancer for the worse. BMC Cancer. 2010; 10:648. [PubMed: 21108799]

8. Kroenke CH, Chen WY, Rosner B, Holmes MD. Weight, weight gain, and survival after breast cancer diagnosis. J Clin Oncol. 2005; 23:1370-1378. [PubMed: 15684320]

9. Topkan E, Parlak C, Selek U. Impact of weight change during the course of concurrent chemoradiation therapy on outcomes in stage IIIB non-small cell lung cancer patients: Retrospective analysis of 425 patients. Int J Radiat Oncol. 2013; 87:697-704.

10. Hess LM, Barakat R, Tian C, Ozols RF, Alberts DS. Weight change during chemotherapy as a potential prognostic factor for stage III epithelial ovarian carcinoma: a Gynecologic Oncology Group study. Gynecol Oncol. 2007; 107:260-265. [PubMed: 17675142]

11. Adams K, Schatzkin A, Harris T, et al. Overweight, obesity, and mortality in a large prospective cohort of persons 50 to 71 years old. N Engl J Med. 2006; 355:763-778. [PubMed: 16926275]

12. Kolotkin R, Meter K, Williams G. Quality of life and obesity. Obes Rev. 2001; 2:219-229. [PubMed: 12119993]

13. Wannamethee S, Shaper A, Walker M. Overweight and obesity and weight change in middle aged men: impact on cardiovascular disease and diabetes. J Epidemiol Community Health. 2005; 59:134-139. [PubMed: 15650145]

14. Kopelman P. Obesity as a medical problem. Nature. 2000; 404:635-643. [PubMed: 10766250]

15. Willett W, Manson J, Stampfer M, et al. Weight, weight change, and coronary heart disease in women. J Am Med Assoc. 1995; 273:461-467.

16. Liu Q, Fayad L, Cabanillas F, et al. Improvement of overall and failure-free survival in stage IV follicular lymphoma: 25 years of treatment experience at The University of Texas M.D. Anderson Cancer Center. J Clin Oncol. 2006; 24:1582-1589. [PubMed: 16575009] 
17. Swenson WT, Wooldridge JE, Lynch CF, Forman-Hoffman VL, Chrischilles E, Link BK. Improved survival of follicular lymphoma patients in the United States. J Clin Oncol. 2005; 23:5019-5026. [PubMed: 15983392]

18. Morton LM, Turner JJ, Cerhan JR, et al. Proposed classification of lymphoid neoplasms for epidemiologic research from the Pathology Working Group of the International Lymphoma Epidemiology Consortium (InterLymph). Blood. 2007; 110:695-708. [PubMed: 17389762]

19. World Health Organization. Global database on body mass index. Accessed January 9, 2015 from: http://apps.who.int/bmi/index.jsp?introPage=intro_3.html

20. Romano PS, Roos LL, Jollis JG. Adapting a clinical comorbidity index for use with ICD-9-CM administrative databases: differing perspectives. J Clin Epidemiol. 1993; 46:1075-1079. [PubMed: 8410092]

21. Savas LS, del Junco DJ, Bastian La, Vernon SW. Mortality ascertainment of women veterans: a comparison of sources of vital status information, 1979-2002. Med Care. 2009; 47:125-128. [PubMed: 19106741]

22. Sohn M-W, Arnold N, Maynard C, Hynes DM. Accuracy and completeness of mortality data in the Department of Veterans Affairs. Popul Health Metr. 2006; 4:2. [PubMed: 16606453]

23. Dafni U. Landmark analysis at the 25-year landmark point. Circ Cardiovasc Qual Outcomes. 2011; 4:363-371. [PubMed: 21586725]

24. Buske C, Hoster E, Dreyling M, Hasford J, Unterhalt M, Hiddemann W. The Follicular Lymphoma International Prognostic Index (FLIPI) separates high-risk from intermediate- or low-risk patients with advanced-stage follicular lymphoma treated front-line with rituximab and the combination of cyclophosphamide, doxorubicin, vinc. Blood. 2006; 108:1504-1508. [PubMed: 16690968]

25. Solal-Céligny P, Roy P, Colombat P, et al. Follicular Lymphoma International Prognostic Index. Blood. 2004; 104:1258-1265. [PubMed: 15126323]

26. Mozaffarian D, Hao T, Rimm EB, Willett WC, Hu FB. Changes in diet and lifestyle and long- term weight gain in women and men. N Engl J Med. 2011; 364:2392-2404. [PubMed: 21696306]

27. Wang Y, Beydoun MA. The obesity epidemic in the United States - gender, age, socioeconomic, racial/ethnic, and geographic characteristics: A systematic review and meta-regression analysis. Epidemiol Rev. 2007; 29:6-28. [PubMed: 17510091]

28. Trotman J, Fournier M, Lamy T, et al. Positron emission tomography-computed tomography (PETCT) after induction therapy is highly predictive of patient outcome in follicular lymphoma: Analysis of PET-CT in a subset of PRIMA trial participants. J Clin Oncol. 2011; 29:3194-3200. [PubMed: 21747087] 


\begin{tabular}{|c|c|}
\hline \multicolumn{2}{|l|}{$\begin{array}{l}\text { Assessed for eligibility } \\
\qquad n=2235\end{array}$} \\
\hline$\downarrow$ & \\
\hline \multicolumn{2}{|l|}{ Excluded $($ Total=1158) } \\
\hline $\begin{array}{l}\text { Other or uncertain diagnosis } \\
\text { CNS involvement at diagnosis } \\
\text { Primary cutaneous } \\
\text { Recurrent FL } \\
\text { No treatment, treated at OSH, or } \\
\text { other treatment }\end{array}$ & $\begin{array}{l}n=87 \\
n=5 \\
n=35 \\
n=27 \\
n=1004\end{array}$ \\
\hline \multicolumn{2}{|l|}{$\begin{array}{c}\text { Total included } \\
n=1077\end{array}$} \\
\hline \multicolumn{2}{|l|}{ Data available for analysis: } \\
\hline Weight at 1 year prior to treatment & $n=634$ \\
\hline Weight at treatment initiation & $\mathrm{n}=896$ \\
\hline $\begin{array}{l}\text { Weight at } 3 \text { months after treatment } \\
\text { initiation }\end{array}$ & $n=896$ \\
\hline $\begin{array}{l}\text { Weight at } 24 \text { months after treatment } \\
\text { initiation }\end{array}$ & $n=583$ \\
\hline Logistic regression & $\mathrm{n}=404$ \\
\hline Landmark analysis & \\
\hline Disease-free survival & $n=796$ \\
\hline Overall, NHL-specific survival & $n=856$ \\
\hline
\end{tabular}

Figure 1.

STROBE Diagram

Abbreviations used: CNS, central nervous system; FL, follicular lymphoma; OSH, outside hospital. 

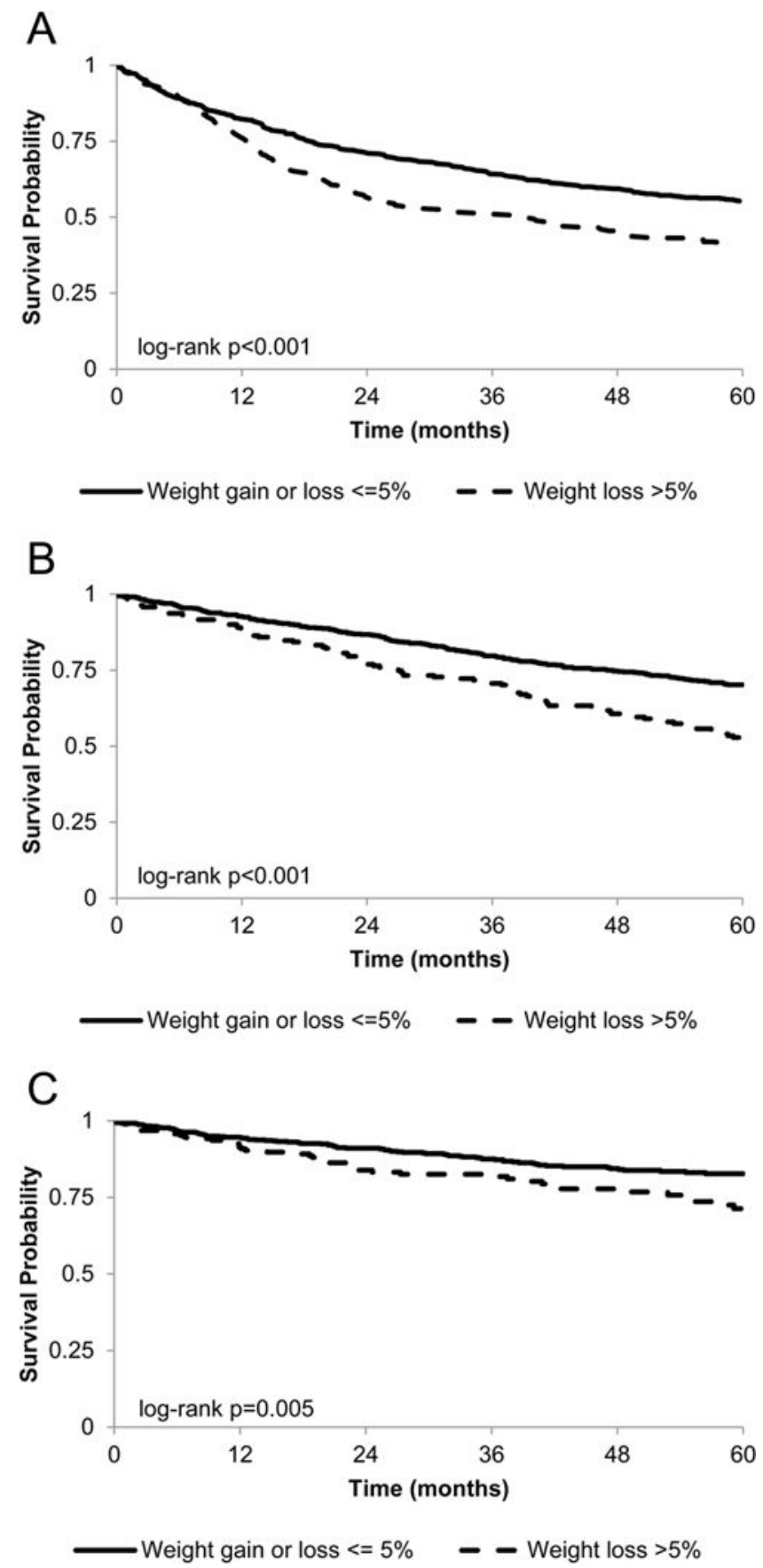

Figure 2.

Kaplan-Meier curves of (A) time to next treatment, (B) overall survival, and (C) NHLspecific survival among US veterans diagnosed with FL from 1998 to 2010 who survived more than 6 months after treatment initiation, stratified by weight change during therapy. Abbreviations used: US, United States; FL, follicular lymphoma; NHL, non-Hodgkin lymphoma. 


\section{Table I}

Demographic and clinical characteristics of US veterans diagnosed with FL from 1998 to 2010 by weight change during treatment.

\begin{tabular}{|c|c|c|c|c|}
\hline \multirow{2}{*}{ Demographic and clinical characteristics } & \multirow{2}{*}{$\frac{\text { Total }}{n=896}$} & \multirow{2}{*}{$\frac{\text { lose } 5 \% \text { or gain }}{n=686}$} & \multirow{2}{*}{$\frac{\text { lose }>5 \%}{n=210}$} & \multirow[t]{2}{*}{ p-value } \\
\hline & & & & \\
\hline Age (mean years) & 63.4 & 62.8 & 65.5 & $<0.001^{*}$ \\
\hline Male Sex $(\%)$ & 96.4 & 96.5 & 96.2 & $0.832 *$ \\
\hline Race $(\%)$ & & & & $0.056^{*}$ \\
\hline White & 89.2 & 88.8 & 90.5 & \\
\hline Black & 8.6 & 9.5 & 5.8 & \\
\hline Other & 2.2 & 1.8 & 3.8 & \\
\hline Co-morbidities (mean Charlson score) & 2.0 & 1.9 & 2.3 & $0.037 \%$ \\
\hline Stage $(\%)$ & & & & $0.650^{\dagger}$ \\
\hline Stage I/II & 24.7 & 25.1 & 23.3 & \\
\hline Stage III/IV & 73.1 & 72.9 & 73.8 & \\
\hline Unknown & 2.2 & 2.0 & 2.9 & \\
\hline LDH $(\%)$ & & & & $0.081^{\dagger}$ \\
\hline Elevated & 26.0 & 24.5 & 31 & \\
\hline Not Elevated & 54.4 & 55.4 & 51 & \\
\hline Unknown & 19.6 & 20.1 & 18.1 & \\
\hline B symptom $(\%)$ & & & & $0.366^{*}$ \\
\hline Yes & 36.9 & 37.8 & 34.3 & \\
\hline No & 59.4 & 58.6 & 61.9 & \\
\hline Unknown & 3.7 & 3.6 & 3.8 & \\
\hline Hemoglobin $(\%)$ & & & & $0.138^{*}$ \\
\hline$<12 \mathrm{~g} / \mathrm{dL}$ & 20.2 & 18.8 & 24.8 & \\
\hline$\geq 12 \mathrm{~g} / \mathrm{dL}$ & 70.9 & 72.4 & 65.7 & \\
\hline Unknown & 8.9 & 8.7 & 9.5 & \\
\hline Year of diagnosis (median) & 2005 & 2006 & 2005 & $0.027 \mathcal{\xi}$ \\
\hline Type of treatment (\%) & & & & $0.002^{*}$ \\
\hline $\mathrm{CHOP}+/-\mathrm{R}$ & 48.5 & 46.1 & 56.7 & \\
\hline $\mathrm{CVP}+/-\mathrm{R}$ & 34.4 & 34.6 & 33.8 & \\
\hline Rituximab only & 17.1 & 19.4 & 9.5 & \\
\hline Height $(\mathrm{cm})$ & 177.2 & 177.5 & 176.3 & $0.039^{t}$ \\
\hline BMI category at treatment initiation (\%) & & & & $0.751^{\dagger}$ \\
\hline$<18.5$ & 1.5 & 1.3 & 1.9 & \\
\hline 18.5 to $<25$ & 28.0 & 27.8 & 26.2 & \\
\hline 25 to $<30$ & 39.2 & 39.1 & 38.6 & \\
\hline 230 & 31.4 & 31.8 & 33.3 & \\
\hline
\end{tabular}


* Chi-square test,

${ }^{\dagger}$ Cochran-Mantel-Haenszel (Nonzero Correlation) test,

$\S_{\text {Wilcoxon test }}$

Abbreviations used: US, United States; FL, follicular lymphoma; LDH, lactate dehydrogenase; CHOP \pm R, cyclophosphamide, doxorubicin, vincristine, and prednisone, with or without rituximab; CVP $\pm \mathrm{R}$, cyclophosphamide, vincristine, and prednisone, with or without rituximab; BMI, body mass index 


\section{Table II}

Univariable and multivariable logistic regression analysis of factors associated with long-term weight gain $>10 \%$ of baseline

\begin{tabular}{|c|c|c|c|c|}
\hline & \multicolumn{2}{|c|}{ Univariable regression analysis } & \multicolumn{2}{|c|}{ Multivariable regression analysi } \\
\hline & OR $(95 \% \mathrm{CI})$ & p-value & OR $(95 \% \mathrm{CI})$ & p-value \\
\hline \multicolumn{5}{|c|}{ Weight change in the year prior to treatment } \\
\hline Loss $5 \%$ or gain & Referent & - & Referent & - \\
\hline Loss $>5 \%$ & 7.38 (3.98 to 13.70$)$ & $<0.001$ & 7.12 (3.54 to 14.33$)$ & $<0.001$ \\
\hline \multicolumn{5}{|c|}{ Weight change during treatment } \\
\hline Loss & Referent & - & Referent & - \\
\hline Gain 0 to $5 \%$ & 1.74 (1.01 to 3.00$)$ & 0.044 & $3.64(1.66$ to 8.00$)$ & 0.001 \\
\hline Gain $>5 \%$ & $6.41(3.45$ to 11.90$)$ & $<0.001$ & 9.11 (3.51 to 23.63 ) & $<0.001$ \\
\hline Age $>60$ years & $0.54(0.34$ to 0.85$)$ & 0.008 & $0.60(0.28$ to 1.25$)$ & 0.171 \\
\hline Stage III/IV & 2.15 (1.13 to 4.10$)$ & 0.02 & $1.81(0.74$ to 4.43$)$ & 0.195 \\
\hline LDH, elevated & 1.69 (1.01 to 2.83$)$ & 0.046 & $1.11(0.51$ to 2.41$)$ & 0.797 \\
\hline Hemoglobin $<12 \mathrm{~g} / \mathrm{dL}$ & 2.67 (1.59 to 4.49$)$ & $<0.001$ & 2.08 ( 0.90 to 4.77$)$ & 0.085 \\
\hline Second-line therapy & $0.83(0.48$ to 1.45$)$ & 0.518 & - & \\
\hline \multicolumn{5}{|c|}{ BMI at treatment initiation } \\
\hline$<25$ & Referent & - & Referent & - \\
\hline 25 to $<30$ & $0.68(0.40$ to 1.16$)$ & 0.157 & $0.78(0.35$ to 1.74$)$ & 0.541 \\
\hline 230 & $0.46(0.25$ to 0.84$)$ & 0.012 & $0.53(0.21$ to 1.30$)$ & 0.162 \\
\hline
\end{tabular}

Abbreviations used: OR, odds ratio; CI; confidence interval; LDH, lactate dehydrogenase; BMI, body mass index. 


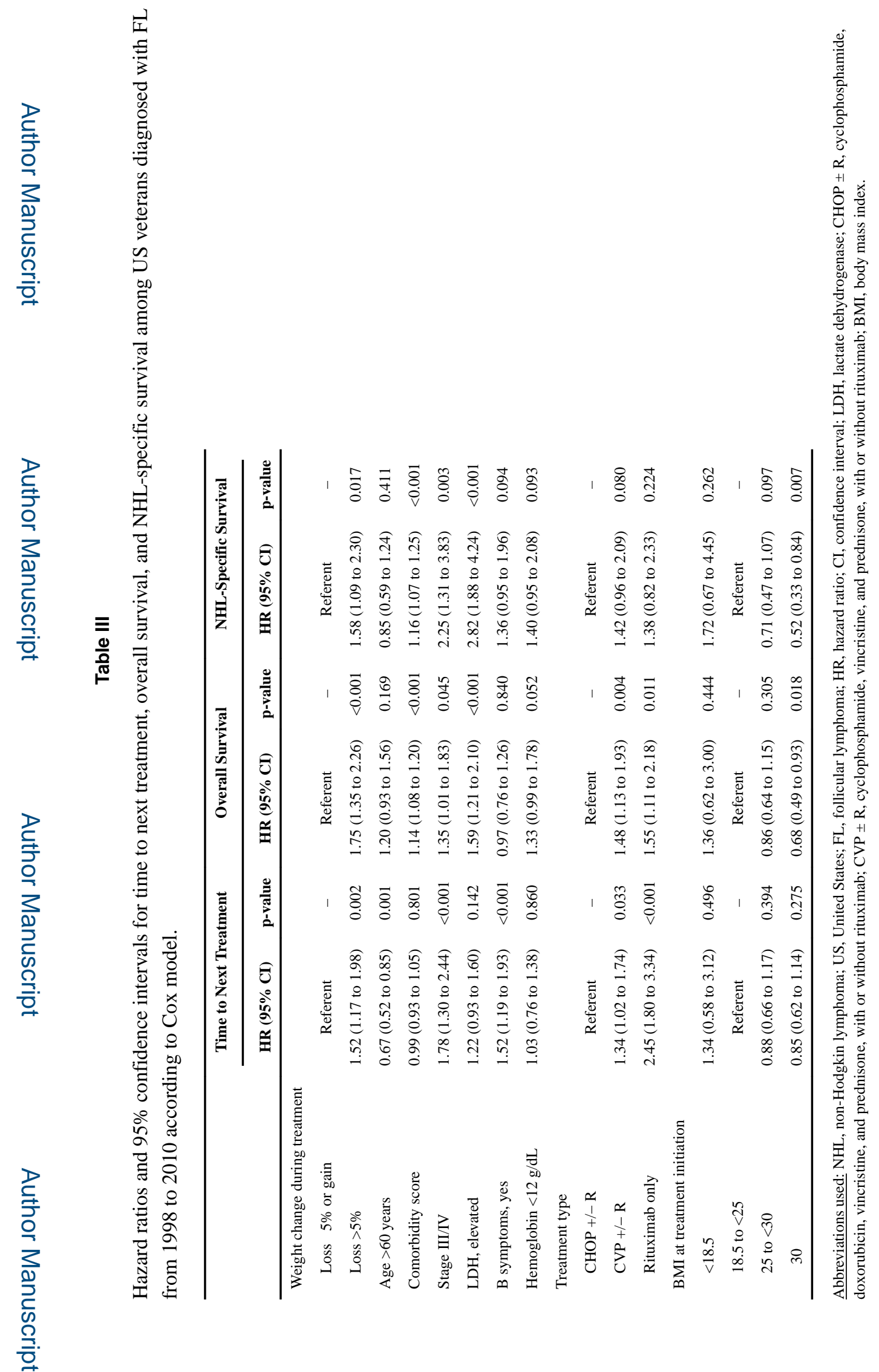

Leuk Lymphoma. Author manuscript; available in PMC 2018 April 01. 